\title{
TIPOS DE AMBLYPYGI (ARACHNIDA: PEDIPALPI) DEPOSITADOS EN EL INSTITUTO DE ECOLOGÍA Y SISTEMÁTICA, LA HABANA, CUBA
}

\author{
Luis F. de Armas \\ Apartado Postal 4327, San Antonio de los Baños, Artemisa 32500, Cuba. luisdearmas1945@gmail.com
}

\section{RESUMEN}

Se listan los tipos porta-nombre (14 holotipos y tres lectotipos), así como 167 paratipos y tres paralectotipos de 21 especies de amblipigios neotropicales depositados en el Instituto de Ecología y Sistemática, La Habana. La mayoría corresponde a especies antillanas (Cuba, República Dominicana, Puerto Rico) y pertenecen a los géneros Charinus Simon, 1892 (Charinidae), Paraphrynus Moreno, 1940 y Phrynus Lamarck, 1801 (Phrynidae). También hay un holotipo de México (Phrynus garridoi Armas, 1995), tres paratipos de Brasil (dos especies de Charinus) y varios paratipos de Costa Rica (Phrynus pseudoparvulus Armas y Víquez, 2001).

Palabras clave: Charinidae, Phrynidae, Charinus, Paraphrynus, Phrynus, taxonomía, Antillas, Región Neotropical.

Title: Types of Amblypygi (Arachnida: Pedipalpi) deposited in the Institute of Ecology and Systematics, Havana, Cuba.

\section{ABSTRACT}

The name-bearing types (14 holotypes, 3 lectotypes), as well as 167 paratypes and 3 paralectotypes belonging to 21 species of Neotropical whip spiders deposited in the Institute of Ecology and Systematics, Havana, are listed. Most of them correspond to Antillean species from Cuba, Dominican Republic, and Puerto Rico, and belong to the genera Charinus Simon, 1892 (Charinidae), Paraphrynus Moreno, 1940 and Phrynus Lamarck, 1801 (Phrynidae). There is also one holotype from Mexico (Phrynus garridoi Armas, 1995), three paratypes from Brazil (two species of Charinus) and several paratypes from Costa Rica (Phrynus pseudoparvulus Armas and Víquez, 2001).

Keywords: Charinidae, Phrynidae, Charinus, Paraphrynus, Phrynus, taxonomy, Antilles, Neotropical Region.

\section{INTRODUCCIÓN}

La casi omnipresencia de los amblipigios en los biotopos cavernícolas y boscosos de los trópicos y subtrópicos del mundo ha contribuido a que sean raros los museos de zoología o de historia natural donde no estén bien representados estos arácnidos. Por supuesto, no en todos han sido adecuadamente estudiados, debido mayormente a los escasos investigadores dedicados a su taxonomía.

Las colecciones aracnológicas del Instituto de Ecología y Sistemática (IES), del Ministerio de Ciencia, Tecnología y Medio Ambiente, La Habana, Cuba, se nutrieron inicialmente (en los primeros años de la década de 1960) de las colecciones de Miguel L. Jaume (1905-1990) y Pelegrín Franganillo Balboa (1873-1955), incorporadas a esta institución, entonces con el nombre de Instituto de Biología de la Academia de Ciencias de Cuba. A partir de 1969, el estudio 
de los amblipigios cubanos se vio beneficiado por el notable incremento de las colecciones aracnológicas del IES (desde esa fecha y hasta 1987 con el nombre de Instituto de Zoología de la Academia de Ciencias de Cuba), los resultados de las expediciones bioespeleológicas cubanorumanas de 1969, 1970 y 1973, así como las revisiones de los géneros Paraphrynus Moreno, 1940 y Phrynus Lamarck, 1801; la primera, realizada por Mullinex (1975); la segunda, por Quintero (1981). A partir de 1987, las colecciones del IES se incrementarían con numerosos especímenes de amblipigios, mayormente antillanos, mexicanos y centroamericanos, incluidos los tipos de un elevado número de especies de los géneros Charinus Simon, 1892 (Charinidae), Paraphrynus Moreno, 1940 y Phrynus Lamarck, 1801 (Phrynidae).

En el caso de los taxones cubanos, es de lamentar la pérdida del catálogo de la colección de Franganillo, así como de una parte de sus ejemplares. Cuando en los primeros años de la década de 1960 esta colección pasó a engrosar los fondos del entonces Instituto de Biología, dicho catálogo ya no existía o no fue entregado junto con los especímenes. Esta situación se hace más lamentable por el hecho de que los frascos únicamente están identificados por el número original de catálogo y carecen de la restante documentación que los identifique (Armas, 1973, 1984, 2014; Quintero, 1983).

Otra es la situación de los tipos de cuatro especies cubanas del género Charinus descritas por Quintero (1983), quien supuestamente los depositó en el Instituto de Zoología de la Academia de Ciencias de Cuba, pero tal acción nunca se realizó y en la actualidad se desconoce el paradero de esos especímenes (Armas, 2004, 2006b).

\section{OBJETIVO}

- Poner en manos de los taxónomos y personas interesadas el inventario de los ejemplares porta-nombre (tipos), así como de los paratipos y paralectotipos depositados en las colecciones aracnológicas del Instituto de Ecología y Sistemática, La Habana, con lo cual se satisface la Recomendación 72F.4 del Código Internacional de Nomenclatura Zoológica (C.I.N.Z., 2000).

\section{MATERIALES Y MÉTODOS}

El presente inventario contiene los tipos primarios (holotipo, lectotipo) y secundarios (paratipos y paralectotipos) de los amblipigios depositados en el Instituto de Ecología y Sistemática (IES), La Habana. En algunas publicaciones y documentos, esta colección aparece identificada por las siglas CZACC (Colecciones Zoológicas de la Academia de Ciencias de Cuba), pero desde finales de la década de 1980 la institución pasó al Ministerio de Ciencia, Tecnología y Medio Ambiente (CITMA). A los efectos de este trabajo, el número de catálogo de cada ejemplar o serie de ejemplares será citado como IES-3.n (donde 3 es el dígito asignado a los arácnidos, excepto ácaros, y "n" corresponde al número consecutivo de la catalogación general).

En la relación de tipos, el orden es solo alfabético.

Características de la colección aracnológica del IES. Todos los arácnidos se hallan en una habitación climatizada permanentemente desde hace más de 30 años. Los especímenes están catalogados y la información correspondiente conservada en soporte digital (base de datos). Los tipos primarios y secundarios están separados del resto. 


\section{RESULTADOS}

\section{CATÁLOGO DE TIPOS}

Familia CHARINIDAE Quintero, 1986

1. Charinus centralis Armas y Ávila Calvo, 2001: 290-291, 292, Fig. 1 A-D, Tabla I. Hembra holotipo (IES-3.3742), Maisinicú, Trinidad, provincia Sancti Spíritus, Cuba, octubre 22, 1985, L. F. Armas, bajo piedras. Paratipos: Cinco machos y dos hembras (IES-3.3739), Maisinicú, Trinidad, 21 de mayo, 1985, L. F. Armas, bajo piedras.

2. Charinus dominicanus Armas y Pérez González, 2001: 52, 62-64, Fig. 14. Hembra holotipo (IES-3.3126), Los Charcos, sección San Rafael, Barahona, provincia Barahona, República Dominicana, agosto 22, 1987, Marcano, Abud, Armas y Lantigua, bajo piedras.

3. Charinus eleonorae Baptista y Giupponi, 2003: 80-84, Figs. 1-14. Macho paratipo (IES3.2971), Gruta Olhos d’Agua, Itacarambi, Minas Gerais, Brasil, [26 de junio, 2001], Giupponi, Baptista et al. Este ejemplar fue declarado en la descripción original (p. 80) como depositado en CZACC; la fecha de recolecta ha sido tomada de la propia descripción original, pues no fue consignada en la documentación que lo acompaña.

4. Charinus perezassoi Armas, 2010: 58, 60, Figs. 2 B-I, 3 F, 4 D, Tabla II. Figs. 2 B-I, 3 F, 4 D, Tabla II. Hembra holotipo (IES-3.3158), finca al final de la carretera 7757, Barrio Los Pollos, Sierra de Guardarraya, Patilla, Puerto Rico, 28 de julio, 2010, L. F. de Armas y A. Pérez Asso, bosque semideciduo antropizado, bajo una vieja lámina de zinc galvanizado, 100 msnm. Paratipos: siete hembras (IES-3.3168 al 3.3170), iguales datos que el holotipo.

5. Charinus tomasmicheli Armas, 2006: 168-169, Figs. 1-4, Tabla I. Macho holotipo (IES3.3735), Cueva Los Manantiales-Tito, Los Manantiales (470 msnm; $21^{\circ} 59^{\prime} \mathrm{N}-80^{\circ}$ 01' O), macizo de Guamuhaya, municipio Manicaragua, provincia Villa Clara, Cuba, 11 de marzo, 2005, T. M. Rodríguez y R. Chaviano, sobre la pared, zona oscura, cerca del arroyo. Paratipos: un macho (IES-3.3741), iguales localidad y recolectores que el holotipo, 15 de octubre, 2004, sobre una acumulación de sedimentos. Una hembra preadulta (IES-3.3740), iguales localidad y recolectores que el holotipo, 16 de octubre, 2004 , en una pequeña oquedad en la pared, zona de penumbra. Un macho adulto, tres hembras preadultas y una hembra juvenil (IES-3.3736), igual localidad que el holotipo, 17 de febrero, 2006, T. M. Rodríguez, zona de penumbra.

6. Charinus troglobius Baptista y Giupponi, 2002: 106-110, Figs. 1-14. Una hembra y un macho paratipos (IES-3.2972 y 3.2973; exMNRJ-9079), Gruna Zé Bastos, Serra 
do Ramalho, Carinhanha, Bahia, Brasil, [28 de junio, 2001], Giupponi, Baptista et al. Ambos paratipos fueron declarados en la descripción original como depositados en el IES; la fecha de recolecta ha sido tomada de la propia descripción original, pues no fue consignada en la documentación que los acompaña.

7. Charinus victori Armas, 2010: 56-58, Figs. 1 A-G, 2 A, 4 D; Tabla I. Macho holotipo (IES-3.3152), Cueva Clara, Sistema Cavernario de Aguas Buenas (18 14' 01' N, - $66^{\circ}$ 06’ 30” O, 250 msnm), Aguas Buenas, Puerto Rico, 23 de Julio, 2010, L. F. Armas, A. Pérez Asso y J. L. Gómez, bajo piedras, zona de penumbras. Paratipos: tres hembras adultas y un macho (IES-3.3164, 3.3166 y 3.3167), iguales datos que el holotipo. Una hembra y un macho (IES-3.3165), proximidades de Cueva La Ventana, Bosque Estatal Río Arriba, Puerto Rico, 18 julio, 2010, L. F. Armas, A. Pérez Asso y A. R. Estrada, bajo piedras, aprox. $300 \mathrm{msnm}$.

Familia PHRYNIDAE Blanchard, 1852

8. Paraphrynus cubensis Quintero, 1983: 7, 10-11, Figs. 2A, 3A, B, 4 D, E, 5 B, 12 A. Hembra lectotipo (designado por Armas, 1984: 3) (IES-3.2231), alrededores de la laguna Ariguanabo, [San Antonio de los Baños], provincia La Habana [actualmente Artemisa], Cuba, febrero de 1972, G. Alayón.

9. Paraphrynus robustus Franganillo, 1930: 120. Macho lectotipo (designado por Armas, 1984: 3), Baracoa, Oriente [actual provincia Guantánamo], Cuba, depositado en la colección de P. Franganillo, frasco No. 664 (IES). Armas (2004: 41) erróneamente lo señaló como una hembra. Paralectotipos: dos macho adultos y una hembra inmadura, iguales datos que el lectotipo.

10. Phrynus alejandroi Armas y Teruel, 2010. Hembra holotipo (IES-3.3161), Punta Verraco, Guayanilla, Puerto Rico, 16 de julio, 2010, L. F. Armas y A. Pérez Asso, bajo piedras, bosque subcostero seco. Paratipos: una hembra y un macho (IES-3.3159), iguales datos que el holotipo. Una hembra y dos juveniles (IES-3.3157), Isla Caja de Muertos, sur de Ponce, Puerto Rico, 24 de julio, 2010, L. F. de Armas y A. Pérez Asso, matorral xeromorfo costero, bajo piedras, 20-40 msnm. Tres hembras, tres machos y un juvenil (IES-3.3160), alrededores del área de acampar, Bosque Estatal Susúa, Puerto Rico, 26 de julio, 2010, L. F. Armas y A. Pérez Asso, bajo piedras, bosque sobre serpentinitas, aproximadamente $400 \mathrm{msnm}$.

11.Phrynus armasi Quintero, 1981: 118, 125, 127, 128, 132-133, 174, Figs. 24-29, mapa 1 (= Phrynus pinarensis Franganillo, 1930; sinonimizada por Armas y Ávila Calvo, 2001: 298. Macho holotipo (IES-3.1260), Cueva El Mudo, Catalina de Güines, La Habana [actual Mayabeque], Cuba, marzo de 1966. Pedipalpo derecho (a partir de la patela), quelícero derecho y placa genital separados del cuerpo; patas fragmentadas. 
12. Phrynus damonidaensis Quintero, 1981: 138-141, Fig. 54-59, 122, 135-137, 164, mapa 1. Hembra holotipo (IES-3.2159), Uvero, El Cobre [actualmente Guamá], Sierra Maestra, provincia Oriente [actualmente Santiago de Cuba], Cuba, 25 de mayo, 1972, L. F. Armas. Este ejemplar tiene el pedipalpo derecho y el quelícero derecho desprendidos; en un frasco adjunto también se conservan los embriones que portaba en el saco ovígero.

13. Phrynus eucharis Armas y Pérez González, 2001: 49, 50, 51-54, 55, 56, 57, 59, 64, Figs. 1, 3 A-D, 4 A, 5 A, 6 A, 7 A, 8. Hembra holotipo (IES-3.3118), El Jabillar, Sánchez, provincia Samaná, República Dominicana, 13 de septiembre, 1987, A. Abud, L.F. Armas, D. Lantigua (bajo piedras). Paratipos: República Dominicana : una hembra, dos machos y un juvenil (IES-3.3117), iguales datos que el holotipo. Siete machos y dos hembras (IES-3.3111 y 3.3113), Palmarito, Samaná, provincia Samaná, septiembre 12, 1987, A. Abud, L.F. Armas, D. Lantigua, bajo piedras. Dos hembras ovígeras (IES-3.3114), Los Yagrumos, sección Los Cacaos, Samaná, septiembre 11, 1987, Abud, Armas, Lantigua, bajo piedras (IES). Una hembra (IES-3.3115), Manuel Chiquito, Samaná, septiembre 12, 1987, Abud, Armas, Lantigua, bajo piedras. Seis hembras y seis machos (IES-3.3121), El Naranjo, Los Haitises, provincia Samaná, septiembre 19, 1987, E. Marcano, A. Abud, L.F. Armas. Seis machos, 14 hembras y tres juveniles (IES-3.3119 y 3.3120), ladera $\mathbf{S}$ de Loma Isabel de Torres, Puerto Plata, provincia Puerto Plata, 25 de febrero de 1999, L. F. Armas, M. Almonte (bajo piedras, 340-380 msnm, bosque secundario). Un macho (IES-3.3116), bajo el puente del río Cumayasa, provincia La Romana, agosto 10, 1987, Marcano, Cicero, Armas, Lantigua. Dos machos, una hembra y dos juveniles (IES3.3112), Bosque de Verón, Higüey, provincia La Altagracia, septiembre 5, 1987, L.F. Armas, A. Abud. Catorce hembras y cinco machos (IES-3.3110), $1 \mathrm{~km}$ después de la entrada a Bayahibe, carretera a Guaraguao, provincia La Altagracia, septiembre 5, 1987, Abud, Armas, Paulino (IES).

14. Phrynus garridoi Armas, 1994: 34-37, Figs. 1-8. Macho holotipo (IES-3.2969), Acapulco, Guerrero, México, noviembre de 1989, O. H. Garrido. Le faltan los últimos segmentos abdominales y el flagelo (tibia y tarsos) de las patas I.

15. Phrynus hispaniolae Armas y Pérez González, 2001: 48, 50, 52, 53, 54-57, 64, Figs. 1, 4 B, 5 B, 6 B, 7 B, 8, 9 A-D. Hembra holotipo (IES-3.3136), Engombe, Distrito Nacional, República Dominicana, 20 de septiembre, 1990, A. J. Abud A. Paratipos: República Dominicana : dos machos (IES-3.3135), iguales datos que el holotipo. Un macho y una hembra (IES-3.3139), vieja carretera Duarte km 32, Pedro Brand, Distrito Nacional, agosto 17, 1987, A. Abud, L. F. Armas, bajo piedras. Un macho (IES-3.3131), ruinas de Engombe, Distrito Nacional, agosto 13, 1987, A. Abud, L. F. Armas, bajo piedras. Una hembra (IES-3.3138), vieja carretera Mella Km 0,1, al Este de Boca Chica, Distrito Nacional, agosto 18, 1987, Abud, Armas, bajo piedras. Tres hembras y tres machos (IES3.3129 y 3.3130), Cueva Ricardo Ramírez, Borbón [actualmente, Cueva No. 4, Reserva El Pomier], San Cristóbal, agosto 26, 1987, L. F. Armas, O. Ramírez, D. Lantigua. 
Dos hembras (IES-3.3127), Majagual, San Cristóbal, septiembre 6, 1987, Abud, Armas, bajo piedras. Un macho (IES-3.3133), La Laguna (carretera a Manaclar km 6), Baní, provincia de Peravia, septiembre 22, 1987, L. F. Armas, P. Ribera, bajo piedras. Dos machos (IES-3.3132), Los Conucos, Guayacanes, provincia San Pedro de Macorís, agosto 10, 1987, E. Marcano, Padre Cicero, L. F. Armas, D. Lantigua, bajo piedras. Un macho (IES-3.3134), Los Conucos, Guayacanes, San Pedro de Macorís, agosto 11, 1987, E. Marcano, L. F. Armas, L. Domínguez, bajo piedra. Una hembra y 2 machos (IES3.3128), Los Bolos (1100 m), Sierra de Neiba, Postrer Río, provincia de Independencia, 14 de abril, 1999, L. F. Armas, bajo piedras. Cuba: una hembra y un macho (IES-3.2629 y 3.2630), arroyo del Campesino, La Esmajagua (ladera Sur del Turquino), Guamá, Santiago de Cuba, 28-29 de junio, 1990, L. F. Armas (bajo piedras, $400 \mathrm{msnm}$ ). Dos machos y dos hembras (IES-3.2623 al 3.2626), Peña Blanca, río Toa, Baracoa, Guantánamo, julio de 1991, Abel Pérez (bajo piedras, 320 msnm). Un macho (IES-3.2622), El Yunque, Baracoa, Guantánamo, 25 de marzo, 1988, L. F. Armas (bajo piedras, 350-600 msnm). Una hembra (IES-3.2627), Cueva de Güiniao, Baracoa, Guantánamo, 21 de noviembre, 1989, A. Ávila y A. A. Socarrás (en la zona oscura).

16. Phrynus kennidae Armas y Pérez González, 2001: 50, 52, 53, 54, 57-59, 64, Figs. 4 C, 5 C, 6 C, 10 A-D, 11. Hembra holotipo (IES-3.3125), Playa del Coco, NO Isla Beata, provincia Pedernales, República Dominicana, 19-20 de marzo, 1999, L. F. Armas, K. Polanco, bajo piedra (IES). Parátipos: un macho adulto y otro juvenil (IES-3.3122), iguales datos que el holotipo. Una hembra (IES-3.3124), igual localidad que el tipo, 20 de marzo, 1999, K. Polanco, L. F. de Armas, bajo corteza, bosque subcostero seco. Un macho juvenil (IES-3.3123), aproximadamente $500 \mathrm{~m} \mathrm{~S}$ Destacamento de la Marina (playa de Punta Beata), Isla Beata, 19 de marzo, 1999, L. F. Armas, K. Polanco, bajo piedra, en bosque seco.

17. Phrynus maesi Armas, 1996: 30, 34-37, Figs. 21-24. Macho paratipo (IES-3.2970), $\mathrm{km} \mathrm{147,5}$ de la carretera que une a Matagalpa con Jinotega (1300 msnm), departamento Jinotega, Nicaragua, 22 de marzo, 1995, J. M. Maes y F. Collantes.

18. Phrynus noeli Armas y Pérez 1994: 7-11, Figs. 1 A-D, 2 A-D, 3 A-C. Hembra holotipo (IES-3.2573), Salón del Caos, Gran Caverna de Santo Tomás, Sierra de Quemado, Viñales, provincia Pinar del Río, Cuba, septiembre de 1992, A. Pérez González, pared, zona oscura; acarreaba siete embriones.

19. Phrynus pinarensis Franganillo, 1930: 92-93. Hembra lectotipo (designada por Armas y Ávila Calvo, 2001: 298), Sierra del Cuzco [Sierra del Rosario], actual provincia de Artemisa, Cuba; depositada en la colección P. Franganillo (IES), con el número 654, sin más datos. 
20. Phrynus pinero Armas y Ávila Calvo, 2001: 294, 297-298, Fig. 2 A-D. Macho holotipo (IES-3.2999), Cueva del Lago, Cerro de la Guanábana, Isla de la Juventud, Cuba, 29 de abril, 1989, A. Ávila Calvo, en la pared.

21. Phrynus pseudoparvulus Armas y Víquez, 2001: 11-15, Figs. 1-2, Tabla I. Tres hembras y dos machos paratipos (IES-3.3743), Heliconias, Bijagua, Upala, provincia Alajuela, Costa Rica, 4 de noviembre, L. F. Armas, bajo troncos podridos, selva húmeda, $700 \mathrm{~m}$. Cuatro hembras y cuatro machos paratipos, (IES-3.3733), Heliconias, Bijagua, Upala, provincia Alajuela, Costa Rica, 3 de noviembre, 2000, L. F. Armas, 720-750 m, bosque húmedo, recolecta nocturna, lloviendo.

\section{CONCLUSIONES}

En la colección aracnológica del Instituto de Ecología y Sistemática están depositados los tipos primarios (porta-nombre) de 17 especies de amblipigios neotropicales (Tablas I y II), la mayoría descritas por este autor y sus colaboradores (Abel Pérez González, Arturo Ávila Calvo, Carlos Víquez, Rolando Teruel), aunque también están representadas dos de las descritas por Franganillo Balboa (1930) y los paratipos de dos especies brasileñas de Charinus descritas por Baptista y Giupponi (2002, 2003). Excepto Phrynus armasi Quintero, 1981, las especies restantes son válidas.

En cuanto a la representatividad por países, en esta colección se hallan los tipos portanombre de nueve especies cuya localidad tipo es Cuba (esto es, de $56 \%$ de las conocidas de este país); cuatro (50\%) de República Dominicana (una compartida con Cuba y otra con Isla Mona, Puerto Rico), tres (60\%) de Puerto Rico (una de ellas compartida con Republica Dominicana) y una de México (Tabla 2). Además, hay numerosos paratipos y tres paralectotipos pertenecientes a 13 especies con localidad tipo en: Cuba (3), República Dominicana (3), Puerto Rico (3), Nicaragua (1), Costa Rica (1) y Brasil (2).

En términos generales, el estado de conservación de todos los especímenes es bueno.

Tabla I. Relación, por géneros, de los tipos de amblipigios depositados en el Instituto de Ecología y Sistemática (IES), La Habana.

\begin{tabular}{|c|c|c|c|c|}
\hline Géneros & Holotipos & Lectotipos & Paratipos & Paralectotipos \\
\hline Charinus & 5 & - & 31 & - \\
\hline Paraphrynus & - & 2 & - & 3 \\
\hline Phrynus & 9 & 1 & 136 & - \\
\hline TOTALES & 14 & 3 & 167 & 3 \\
\hline
\end{tabular}


Tabla II. Relación, por países, de la cantidad de especies de amblipigios cuyos tipos portanombre y tipos secundarios (paratipos y paralectotipos) están depositados en el Instituto de Ecología y Sistemática (IES), La Habana.

\begin{tabular}{|c|c|c|c|c|}
\hline País & Holotipos & Lectotipos & Paratipos & Paralectotipos \\
\hline Cuba & 6 & 3 & $3^{\text {a }}$ & 1 \\
\hline $\begin{array}{c}\text { República } \\
\text { Dominicana }\end{array}$ & 4 & - & 3 & - \\
\hline Puerto Rico & 3 & - & 3 & - \\
\hline México & 1 & - & - & - \\
\hline Nicaragua & - & - & 1 & - \\
\hline Costa Rica & - & - & 1 & 1 \\
\hline Brasil & - & - & 12 & - \\
\hline TOTALES & 14 & 3 & & 2 \\
\hline
\end{tabular}

a: Una especie compartida con República Dominicana.

-En el caso de los tipos portanombre, las especies distribuidas en más de un país han sido asignadas únicamente a aquel donde está su localidad tipo; no así en cuanto a los tipos secundarios.

\section{LITERATURA CITADA}

Armas, L. F. de. 1973. Tipos de las colecciones escorpiológicas P. Franganillo y Universidad de La Habana (Arachnida: Scorpionida). Poeyana, 101: 1-18.

Armas, L. F. de. 1984. Tipos de Arachnida depositados en el Instituto de Zoología de la Academia de Ciencias de Cuba. I. Amblypygi, Opiliones, Ricinulei, Scorpiones, Schizomida y Uropygi. Poeyana, 284: 1-11.

Armas, L. F. de. 1994. Nueva especie de Phrynus (Amblypygi: Phrynidae) del estado de Guerrero, México. AvaCient 9: 34-37.

Armas, L. F. de. 1996. Nuevos Phrynus de México y Nicaragua, con la descripción complementaria de P. garridoi Armas (Amblypygi: Phrynidae). Revista Nicaragüense de Entomología, 33: 21-37.

Armas, L. F. de. 2004. Arácnidos de República Dominicana. Palpigradi, Schizomida, Solifugae y Thelyphonida (Chelicerata: Arachnida). Revista Ibérica de Aracnología, vol. Especial Monogr., 2: 1-64.

Armas, L. F. de. 2006a. Nueva especie de Charinus Simon, 1892 (Amblypygi: Charinidae) de Cuba central. Revista Ibérica de Aracnología, 13: 167-170.

Armas, L. F. de. 2006b. Sinopsis de los amblipígidos antillanos (Arachnida: Amblypygi). Boletín de la Sociedad Entomológica Aragonesa, 38: 223-245. 
Armas, L. F. de. 2010. Nuevos arácnidos de Puerto Rico (Arachnida: Amblypygi, Araneae, Opiliones, Parasitiformes, Schizomida, Scorpiones). Boletín de la Sociedad Entomológica Aragonesa, 47: 55-64.

Armas, L. F. de. 2014. Los amblipigios de Cuba (Arachnida: Amblypygi). Revista Ibérica de Aracnología, 24: 29-51.

Armas, L. F. de y A. Ávila Calvo. 2001. Dos nuevos amblipígidos de Cuba, con nuevos sinónimos y registros (Arachnida: Amblypygi). Anales de la Escuela Nacional de Ciencias Biológicas, México, 46(3): 289-303.

Armas, L. F. de y A. Pérez [González]. 1994. Description of the first troglobitic species of the genus Phrynus (Amblypygi: Phrynidae) from Cuba. Avicennia, 1: 7-11.

Armas, L. F. de y A. Pérez González. 2001. Los amblipígidos de República Dominicana (Arachnida: Amblypygi). Revista Ibérica de Aracnología, 3: 47-66.

Armas, L. F. de y R. Teruel. 2010. Nueva especie de Phrynus Lamarck, 1801 (Amblypygi: Phrynidae) de Puerto Rico. Boletín de la Sociedad Entomológica Aragonesa, 47: 127-130.

Armas, L, F. de y C. Viquez. 2001. Nueva especie de Phrynus (Amblypygi: Phrynidae) de Costa Rica. Revista Ibérica de Aracnología, 4:11-15.

Baptista, R. L. C. y A. P. L. Giupponi. 2002. A new troglomorphic Charinus from Brazil. Revista Ibérica de Aracnología, 6: 105-110.

Baptista, R. L. C. y A. P. L. Giupponi. 2003. A new troglomorphic Charinus from Minas Gerais, Brazil (Arachnida: Amblypygi: Charinidae). Revista Ibérica de Aracnología, 7: 79-84.

Comisión Internacional de Nomenclatura Zoológica (C.I.N.Z.). 2000. Código internacional de nomenclatura zoológica. 4ta edición. International Trust for Zoological Nomenclature, Madrid. i-xxix +156 pp.

Franganillo Balboa, P. 1930. Más arácnidos nuevos de la Isla de Cuba. Memorias del Instituto Nacional de Investigaciones Científicas y Museo de Historia Natural, La Habana, 1: 45-99.

Mullinex, C. L. 1975. Revision of Paraphrynus Moreno (Amblypygida: Phrynidae) for North America and the Antilles. Occasional Papers of the California Academy of Sciences, 116: $1-80$.

Quintero, D., Jr. 1981.The amblypygid genus Phrynus in the Americas (Amblypygi, Phrynidae). Journal of Arachnology, 9(2): 117-166.

Quintero, D., Jr. 1983. Revision of the amblypygid spiders of Cuba and their relationships with the Caribbean and continental American amblypygid fauna. Studies on the Fauna of Curacao and other Caribbean Islands, 65: 1-54.

[Recibido: 20 de abril, 2014. Aceptado para publicación: 30 de Julio, 2014] 